\title{
Vertrauen ist gut, Kontrolle ist besser
}

\author{
WISSENSCHAFT ERKLÄRT: KONFIDENZINTERVALLE Studienergebnissen sollte man \\ nicht blind vertrauen. Man sollte nach Faktoren Ausschau halten, die zeigen, wie \\ sicher die Resultate tatsächlich sind. Konfidenzintervalle geben Aufschluss darüber, \\ wie hoch die Genauigkeit einer Studie ist.
}

$S$

elbst sehr gut durchgeführte Studien geben uns mit ihren Ergebnissen nur Hinweise und keine vollständigen Antworten auf unsere Fragen. Da sich Ergebnisse einer Studie immer auf eine Stichprobe aller Patienten beziehen, unterliegen sie einer statistischen Unsicherheit. Doch wie sicher oder wie unsicher sind die Ergebnisse? Hat das Vertrauen in Studienergebnisse etwas mit Zufall und Wahrscheinlichkeiten zu tun? Ja, das hat es! Eine der anerkanntesten Methoden zur Bestimmung von Unsicherheiten zum Beispiel bei Messungen sind die Konfidenzintervalle. Konfidenz bedeutet so viel wie Vertrauen, deshalb nennt man ein Konfidenzintervall auch Vertrauensbereich. Wie passen aber Vertrauen und Unsicherheit zusammen? Und wie wichtig ist das alles für den physiotherapeutischen Alltag?

Die Genauigkeit von Messergebnissen bestimmen > Mithilfe von Konfidenzintervallen beschreiben Wissenschaftler die Genauigkeit (positiv ausgedrückt) beziehungsweise die Ungenauigkeit (negativ ausgedrückt) von Untersuchungsergebnissen. Letztere nennt man in der Statistik auch Schätzung. Da jede Untersuchung, die sich auf eine Auswahl von Probanden bezieht, den Effekt aller Studienteilnehmer nur schätzen kann.

Konfidenzintervalle bestehen aus einer oberen und einer unteren Konfidenzgrenze (॰ Abb. 1). Die Konfidenzintervalle beschreiben Messergebnisse sowie deren Genauigkeit und gehören deshalb zur deskriptiven Statistik. In den meisten Fällen geben Forscher ein Konfidenzintervall von $95 \%$ an. Das bedeutet beispielsweise für den Mittelwert, dass der angegebene Konfidenzintervall (KI) den wahren Mittelwert in der Bevölkerung mit 95\%-iger

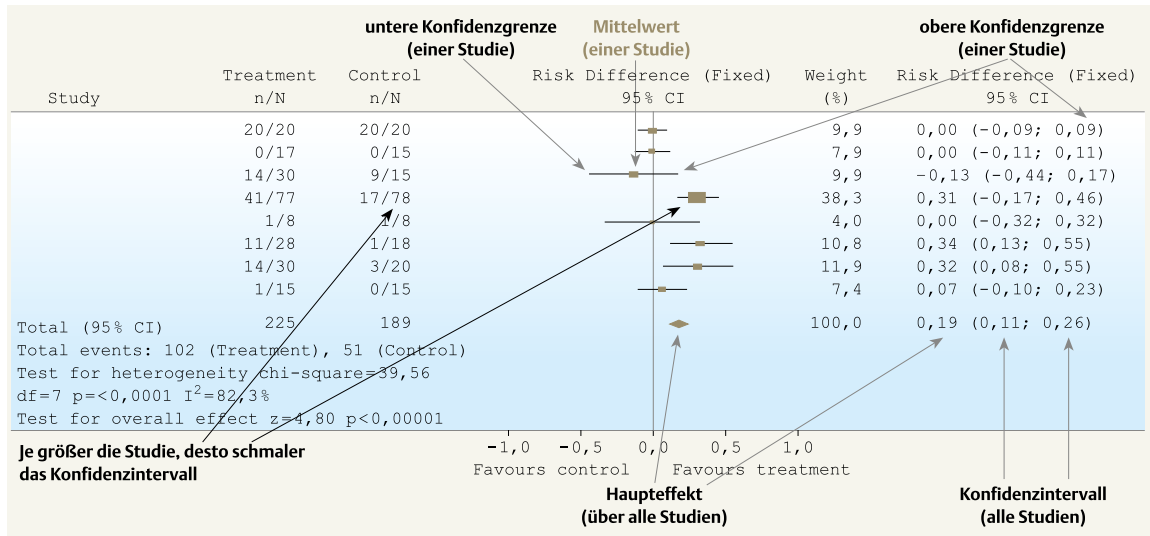

Abb. 1 Konfidenzintervalle in einer Metaanalyse

Wahrscheinlichkeit überlappt. Es gibt verschiedene Schreibweisen für einen Mittelwert. Hier am Beispiel von $8,2 \mathrm{~mm}$ :

$>8,2 \mathrm{~mm} 95 \% \mathrm{KI}(6,5 \ldots 9,8)$ oder

$>8,2 \mathrm{~mm} 95 \% \mathrm{Cl}(6,5 \ldots 9,8)$ oder

$>8,2 \mathrm{~mm} 95 \% \mathrm{KI}(6,5 ; 9,8)$ oder

$>8,2 \mathrm{~mm} 95 \% \mathrm{Cl}[6.5,9.8]$

Eine falsche Schreibweise wäre „95\% KI: von 6,5 bis $9,8 “$.

\section{Größe der Stichprobe hat Einfluss auf} das Konfidenzintervall > Da man mithilfe von Konfidenzintervallen nicht nur Ergebnisse beschreibt (deskriptive Statistik), sondern die Wissenschaftler mit innen zusätzlich statistische Schlussfolgerungen ziehen, gehören die Konfidenzintervalle auch zur sogenannten induktiven Statistik. Zum Beispiel könnten sich Forscher fragen, ob Patienten, die Ausdauertraining absolvieren, zufriedener sind als Patienten, die Dehnungsübungen machen. Ist sich die Forschergruppe nicht einig, teilen sie die Patienten in zwei Gruppen ein. Die eine hat als Schwerpunkt Ausdauertraining und die andere Gruppe beinhaltet Patienten, die mit Dehnen ihre Beweglichkeit verbessern. Das Beispiel ist methodisch nicht ganz einwandfrei, aber sehr praxisnah.

Als Zielparameter für diese Studie legen die Forscher die Patientenzufriedenheit (PZ) fest, die sie nach vier Wochen auf einer intervallskalierten Skala von 0 bis 10 messen. Das Ergebnis wird mit einem Mittelwert und dem dazugehörigen Konfidenzintervall dargestellt: > PZ-Gruppe 1 = 8,2 95\% KI $(6,5 . . .9,8)$

> PZ-Gruppe 2 = 7,6 95\% KI $(5,9 . . .9,2)$ Gruppe 1 zeigt im Vergleich zu Gruppe 2 eine höhere Patientenzufriedenheit. Könnte dies nicht Zufall sein? Ja, es könnte, weil sich die beiden Konfidenzintervalle überlappen (9,2 ist in „KI 6,5...9,8“ enthalten). Das Ergebnis ist nicht eindeutig. Allgemein gilt, dass bei einer hohen Zahl an Patienten in einer Studie der Konfidenzintervall schmaler wird, und je größer die Stichprobe der Patienten ist, desto präziser ist der bestimmte Effekt.

Konfidenzintervalle sind mittlerweile sehr verbreitet, einige Zeitschriften verpflichten ihre Autoren sogar dazu, diese anzugeben.

Prof. Dr. Jan Mehrholz 